\title{
The effect of menstrual phase and hormonal contraception on successful bilateral placement of the Essure micro-insert tubal coil
}

\author{
Christine D. Lett • John A. Thiel
}

Received: 11 July 2008 / Accepted: 15 October 2008 / Published online: 6 November 2008

(C) Springer-Verlag 2008

\begin{abstract}
The objective of this study was to determine the effect of menstrual phase and preoperative hormonal contraception on successful bilateral placement of the Essure micro-insert tubal coil. It is a retrospective review (Canadian Task Force classification II-2) which was completed in an ambulatory women's health center in the Regina Qu'Appelle Health Region. Women of reproductive age presenting with a request for permanent contraception using the hysteroscopic sterilization with the Essure microinsert coil were enrolled. The main outcome measure was the successful bilateral placement of the Essure micro-insert tubal coil defined as bilateral visualization of the tubal ostia and cannulation with the Essure micro-insert coil. Eightyone of 84 patients $(96 \%)$ had successful visualization of the tubal ostia with bilateral placement achieved in $78 / 81$ $(96 \%)$. There was no difference $(p=959)$ in success rate based on the menstrual phase nor was there a difference between patients using hormonal contraception compared to patients using non-hormonal contraception $(p=0.557)$. Successful bilateral placement of the Essure micro-insert tubal coil can be completed in all phases of the menstrual cycle, without endometrial suppression by hormonal contraceptives.
\end{abstract}

Keywords Sterilization · Hysteroscopy ·

Contraceptive devices · Menstrual cycle · Fallopian tubes .

Retrospective study

C. D. Lett $\cdot$ J. A. Thiel $(\triangle)$

Department of Obstetrics and Gynecology,

Regina Qu'Appelle Health Region,

100-2631 28th Avenue,

Regina, Saskatchewan, Canada S4S 6X3

e-mail: drjthiel@aol.com

\section{Introduction}

The Essure micro-insert coil tubal sterilization procedure is becoming increasingly common as women seek to obtain safe and effective methods of permanent contraception. It provides reliable permanent contraception with none of the risk associated with laparoscopic tubal sterilization. As more physicians adopt the Essure method for sterilization, they continue to look for techniques to improve the rate of successful bilateral coil placement on the first attempt. The reported bilateral placement rate ranges from $81 \%$ [1] to $98 \%$ [2]. It has been suggested that the procedure be completed in the follicular phase to improve visualization of the tubal ostia, and thus the successful placement of the coils [3-5]. Alternatively, hormonal endometrial suppression with the oral contraceptive pill, a progestin, or leuprolide before the procedure has been proposed to improve visualization [6]. Attempting to time the procedure according to the follicular phase of the menstrual cycle can create scheduling problems for physicians who perform the procedure outside of a private office facility. It can also be difficult to arrange a time for the procedure in women who have irregular menstrual cycles. In addition, the use of suppressive hormonal therapy may be problematic for women who have contraindications to such medications or for women who are choosing permanent sterilization to avoid the use of hormonal contraceptives.

To date, the effects of cycle timing or endometrial suppression on improving successful Essure coil placement have not been investigated. The objective of the present study was to determine the effect of the menstrual cycle phase and preoperative endometrial suppression using hormonal contraceptives on the successful bilateral placement of the Essure micro-insert coil. We hypothesized that menstrual phase timing or endometrial suppression with a 
hormonal contraceptive would improve our ability to place the Essure coils successfully on the first attempt.

\section{Materials and methods}

The clinical charts of women who underwent Essure hysteroscopic sterilization procedures from December 1, 2005 to December 31, 2006 in the ambulatory outpatient clinic at Regina General Hospital, Canada, were reviewed retrospectively (Canadian Task Force classification II-2). Ethical approval was obtained from the Regina Qu'Appelle Health Region Ethics Review Board.

In all cases, placement of the Essure micro-insert coils was completed in an ambulatory setting using the technique as described by Kerin et al. [2]. In our center, all patients receive indomethacin $100 \mathrm{mg}$ per rectum $1 \mathrm{~h}$ before the procedure and are administered either intravenous conscious sedation with fentanyl (Abbott Laboratories, Ltd., Toronto, Canada) $2 \mu \mathrm{g} / \mathrm{kg}$ body weight and diazepam 2.5 mg (Pharmacia Canada, Mississauga, Canada) or oral analgesia with oxycodone (Sandoz Canada Inc., Boucherville, Canada) $10 \mathrm{mg}$.

Direct visualization of the tubal ostium was accomplished using a $2.7-\mathrm{mm} 12^{\circ}$ diagnostic telescope in a $5.5 \mathrm{~mm}$ single channel operative hysteroscope (ACMI, Toronto, Canada) with normal saline as the distending medium. The use of the Niagara fluid pump (Circon ACMI, Stamford, CT, USA) ensured that the saline was delivered at a constant pressure. The usual pressure setting selected was $200 \mathrm{mmHg}$ which could be further increased to $300 \mathrm{mmHg}$ if the endometrium was thickened.

The primary outcome measure was successful bilateral placement of the Essure micro-insert tubal coil defined as bilateral visualization of the tubal ostia and cannulation with the Essure micro-insert coil on the first attempt. Charts were excluded if the patient was returning for a repeat procedure or if she only required unilateral coil placement.

Confirmation of coil position was completed 3 months after the procedure using volume contrast ultrasonography as previously described $[7,8]$. If the ultrasound examination was not successful in identifying appropriate coil position, hysterosalpingography (HSG) was used to demonstrate tubal occlusion. Patients were instructed to use alternative contraception until confirmation of successful coil placement was completed.

The cohort was divided based on menstrual phase to determine its effect on successful Essure coil placement. Patients were grouped into three categories based on the interval from their last menstrual period (LMP): phase A (0-10 days), phase B (11-20 days), and phase C (>20 days). The cohort was also grouped based on method of contraception (hormonal or non-hormonal) to determine the effect of hormonal suppression of the endometrium on successful Essure coil placement.

Statistical analysis with SPSS 14.0 was completed using $\chi^{2}$ for categorical data and the Student's $t$ test for continuous data. Statistical significance was set at $p<0.05$.

\section{Results}

The charts of 84 women who underwent the Essure microinsert hysteroscopic sterilization procedure during the study period were reviewed. Patient characteristics are summarized in Table 1. The mean age of the patients undergoing the Essure procedure was 37.1 years $( \pm \mathrm{SD} 5.9)$, the mean gravidity and parity were $2.2( \pm$ SD 1.3$)$ and $2.0( \pm$ SD 1.1$)$, respectively. Conscious sedation was used in 58 of the 84 women $(69 \%)$ and oral analgesia was used in 26 of the $84(31 \%)$.

Thirty women $(36 \%)$ were in phase A (day $0-10$ from LMP), 22 (26\%) were in phase B (day 11-20), and 32 (38\%) were in phase C (>day 20) (Table 1). Forty-two women $(50 \%)$ were using hormonal contraception at the time of the procedure. Forty-two women (50\%) were using non-hormonal methods.

Both tubal ostia were visualized successfully in $96 \%$ of patients (Table 2). Once visualization had occurred, successful bilateral cannulation followed in $96 \%$ of patients. One patient (1\%) was lost to follow-up and thus medical imaging reports were available in 83 of the 84 charts (99\%). Of the 83

Table 1 Patient characteristics

\begin{tabular}{ll}
\hline Baseline characteristics, mean (range) & \\
Age (years) & $37.1(23-53)$ \\
Gravidity & $2.2(0-6)$ \\
Parity & $2.0(0-5)$ \\
Weight $(\mathrm{kg})$ & $72.0(49.2-131)$ \\
Cycle day & $23.1(1-66)$ \\
Method of contraception, number (\%) & \\
Hormonal & $42(50 \%)$ \\
Oral contraceptive pill & 31 \\
Evra patch & 4 \\
Depo-Provera & 6 \\
Mirena & 1 \\
Non-hormonal & $42(50 \%)$ \\
Barrier & 24 \\
Other & 4 \\
None & 11 \\
Not recorded & 3 \\
Cycle day & Number $(\%)$ \\
$0-10^{a}$ & $30(36 \%)$ \\
$11-20$ & $22(26 \%)$ \\
$>20$ & $32(38 \%)$ \\
Method of sedation, number $(\%)$ & \\
Oral & $26(31 \%)$ \\
Intravenous & $58(69 \%)$ \\
\hline
\end{tabular}

${ }^{a}$ Includes eight patients amenorrheic on hormonal suppression 
Table 2 Successful visualization of the tubal ostia and successful bilateral cannulation with the Essure micro-insert coil

${ }^{\text {a }}$ Seven charts with imaging unavailable, patients lost to follow-up

\begin{tabular}{llll}
\hline & $\begin{array}{l}\text { Successful } \\
\text { visualization }(\%)\end{array}$ & $\begin{array}{l}\text { Successful cannulation } \\
\text { after visualization }(\%)\end{array}$ & $\begin{array}{l}\text { Successful placement confirmed } \\
\text { by medical imaging }(\%) n=71^{\text {a }}\end{array}$ \\
\hline All patients & $81 / 84(96 \%)$ & $78 / 81(96 \%)$ & $68 / 71(96 \%)$ \\
Cycle day & $p=0.959$ & $p=0.955$ & $p=0.307$ \\
Day 1-10 & $29 / 30$ & $28 / 29$ & $22 / 24$ \\
Day 11-20 & $21 / 22$ & $20 / 21$ & $17 / 18$ \\
Day >20 & $31 / 32$ & $30 / 31$ & $29 / 29$ \\
Hormonal contraception & $p=0.557$ & $p=0.542$ & $p=0.506$ \\
Yes & $40 / 42$ & $38 / 40$ & $32 / 34$ \\
No & $51 / 42$ & $40 / 41$ & $46 / 37$ \\
\hline
\end{tabular}

cases, successful bilateral placement was demonstrated by ultrasonography or HSG in $96 \%$. There was no difference in success rates (visualization, cannulation, visualization of proper coil placement) among cycle phases $(p=0.959, p=$ $0.955, p=0.307$ ) or between women using hormonal versus non-hormonal methods of contraception $(p=0.557, p=0.542$, $p=0.506$ ).

Failure to visualize the tubal ostia occurred in three patients. In one case, the left ostium was not visualized, but the right ostium was visualized and successfully cannulated. The patient returned 4 months later for a repeat Essure procedure on the left, which was then successful. The other two failures to visualize the ostia were related to difficulty accessing the endometrial cavity. One failure to access the cavity was secondary to cervical stenosis in a nulligravid patient. The other procedure was abandoned because of a fundal perforation in a patient who was amenorrheic on Depo-Provera.

Three failures to cannulate one or both ostia were recorded. In all three cases, the tubal ostia were initially visualized. In two cases, an HSG subsequently documented bilateral tubal occlusion without placement of the coil. The third patient with failed bilateral cannulation was suspected to have bilateral tubal spasm at the time of the procedure. She returned 2 months later for a repeat procedure, which was then successful.

\section{Discussion}

Menstrual phase timing and hormonal endometrial suppression did not improve successful bilateral placement of the Essure micro-insert coils in this retrospective study. This finding is consistent with suggestions from previous studies that menstrual phase [9] and preoperative hormonal manipulation [10] did not appear to improve bilateral placement rates.

In 2001, the first clinical study of Essure reported a bilateral placement rate of $86 \%$ [11]. Successful bilateral placement improved during phase II and phase III trials to $88 \%$ [9] and 92\% [3], respectively. The improvement was attributed, in part, to operator experience [9]. After the introduction of the coil catheter delivery system, a $98 \%$ bilateral placement rate was reported in a cohort of 102 women [2]. The improvement in successful placement was attributed to the improved coil catheter delivery system [2]. The two failures reported in this study were failures to cannulate the ostia after adequate visualization. Difficulty accessing the endometrial cavity was not reported. In addition, women were excluded if they were nulliparous or at risk for tubal disease (previous ectopic pregnancy, previous pelvic inflammatory disease, previous salpingectomy, chronic pelvic pain).

Successful visualization of the tubal ostia was demonstrated in $96 \%$ of patients, which included nulliparas and those at risk for tubal disease. In two of the three failures to visualize, there was a complication that prevented entry into the uterine cavity. Once visualization had occurred, successful cannulation followed in $96 \%$ of patients. One failure to cannulate was attributed to bilateral tubal spasm which has been documented to prevent successful hysteroscopic sterilization in up to $4 \%$ of patients [12]. The two other failures were due to pre-existing tubal occlusion as demonstrated by HSG after the attempted Essure coil placement.

The successful visualization of the tubal ostia in women with a thicker, non-suppressed luteal endometrium may have been related to the pressure used to deliver the saline distention medium. The use of the Niagara fluid pump (Circon ACMI) ensured that the saline was delivered at a constant pressure throughout the procedure. The pressure could also be slightly increased when a thickened endometrium was encountered. The initial pressure setting selected was $200 \mathrm{mmHg}$ which was further increased to $300 \mathrm{mmHg}$ if the endometrium was thickened. The $200 \mathrm{mmHg}$ pressure is higher than has been previously reported [5, 13]. Anecdotally, the resultant increase in uterine distention can result in some minimal baroceptor-related discomfort for the patient; however, with adequate analgesia, this very transient effect is well tolerated. Since the procedure time is short, the increase in pressure does not result in significant intravasation of saline. The higher pressure further flattens the endometrium and distends the tubal ostia, thus ensuring adequate visualization. The improved visualization and 
distended proximal tube makes for an easier and faster placement of the Essure coil. The shorter procedure time is also related in part to operator experience which, as was previously noted, will improve the bilateral placement rate.

The potential risk of placing the Essure coils in the luteal phase of the menstrual cycle in a patient with an undetected pregnancy is extremely small. In one recent study, only seven of approximately 50,000 patients $(0.0000014 \%)$ undergoing the procedure were suspected to have been pregnant at the time of the coil placement [14]. This statistic is lower that the $0.0003 \%$ risk noted in the CREST study [15]. The maximum efficiency of human reproduction has been estimated to be as high as $30 \%$ per cycle with a significant number of these conceptions being occult or ending in spontaneous abortion [16]. One could postulate that the risk of having the procedure delayed in order to time the coil placement in the follicular phase would result in more pregnancies in the intervening month secondary to failure of the contraceptive method. There is no recommendation that patients undergo sterilization by laparoscopy or laparotomy only in the follicular phase, in spite of the fact that the risk of luteal phase pregnancy was discussed in the CREST data [15]. We conclude that the risk of luteal pregnancy is outweighed by the risk of unintended pregnancy if the procedure is delayed. Our standard operating procedure is to counsel women accordingly if they arrive for their procedure in the latter half of their cycle. Only $13 \%$ of the patients in our study were without contraception and thus were at greater risk of an unintended pregnancy if their procedure was completed in the luteal phase. Women exposed to unprotected intercourse would be offered the opportunity to reschedule their procedure if they were concerned that the risk of pregnancy was too high. No procedure failures associated with a luteal phase pregnancy were noted; however, the present study was not adequately powered to detect a difference given the very small risk.

One limitation of this study is that it was not adequately powered to detect a small difference in successful bilateral placement rates. Our sample size calculation was based on a lower rate of successful placement of $81 \%$. Our overall success rate was much higher than this, at $96 \%$. Our study was stopped at 42 patients per arm given that there were no differences noted in any of the groups followed. Given that our initial placement rate was higher, we did not feel that enrolling a further 13 patients per group would demonstrate any significant differences. In fact, approximately 2,000 women would be required to detect a small difference in success rates (from $96 \%$ to $98 \%$ ) based on menstrual phase or endometrial suppression. These numbers could be achieved by conducting a multicenter randomized trial. However, even if such a small difference were present, it may not be clinically relevant and thus may not justify mandatory endometrial suppression or menstrual cycle timing for all patients.
The rate of successful bilateral Essure micro-insert coil placement on first attempt was very high. Success rates were not improved by performing the procedure in the early follicular phase or by using preoperative hormonal endometrial suppression. Therefore, we suggest that physicians experienced in placing the Essure coils and using adequate pressures for uterine distension need not initiate hormonal suppression prior to the procedure, particularly in patients who have chosen to use alternative methods of contraception. The Essure procedure can then be booked without regard for the phase of the menstrual cycle, and after appropriate counseling can be scheduled at a time convenient to both surgeon and patient.

\section{References}

1. Duffy S, Marsh F, Rogerson L et al (2005) Female sterilisation: a cohort controlled comparative study of ESSURE versus laparoscopic sterilization. BJOG 112:1522-1528

2. Kerin JF, Munday DN, Ritossa MG et al (2004) Essure hysteroscopic sterilization: results based on utilizing a new coil catheter delivery system. J Am Assoc Gynecol Laparosc 11:388-393

3. Cooper JM, Carignan C, Cher D et al (2003) Microinsert nonincisional hysteroscopic sterilization. Obstet Gynecol 102:59-67

4. Cooper JM (1992) Hysteroscopic sterilization. Clin Obstet Gynecol 35:282-298

5. Chern B, Siow A (2005) Initial Asian experience in hysteroscopic sterilization using the Essure permanent birth control device. BJOG 112(9):1322-1327

6. Levy B, Dobbins B, Greenberg JA (2005) Protocols for in-office hysteroscopic sterilization: a roundtable discussion Available via http:// www.obgmanagement.com OBG Management, Supplement, October

7. Thiel JA, Suchet IB, Lortie K (2005) Confirmation of Essure microinsert tubal coil placement with conventional and volumecontrast imaging three-dimensional ultrasound. Fertil Steril 84:504-508

8. Veersema S, Vleugels MPH, Timmermans A et al (2005) Followup of successful bilateral placement of Essure microinserts with ultrasound. Fertil Steril 84:1733-1736

9. Kerin JF, Cooper JM, Price T et al (2003) Hysteroscopic sterilization using a micro-insert device: results of a multicentre Phase II study. Hum Reprod 18:1223-1230

10. Nichols M, Carter JF, Fylstra DL (2006) A comparative study of hysteroscopic sterilization performed in-office versus a hospital operating room. J Minim Invasive Gynecol 13:447-450

11. Kerin JF, Carignan CS, Cher D (2001) The safety and effectiveness of a new hysteroscopic method for permanent birth control: results of the first Essure clinical study. Aust NZJ Obstet Gynaecol 41:364-370

12. Cooper JM, Rigberg HS, Houck R et al (1985) Incidence, significance and remission of tubal spasm during attempted hysteroscopic tubal sterilization. J Reprod Med 30:39-42

13. Magos A, Chapman L (2004) Hysteroscopic tubal sterilization. Obstet Gynecol Clin N Am 31:705-719

14. Levy B, Levie MD, Childers ME (2007) A summary of reported pregnancies after hysteroscopic sterilization. J Minimal Invasive Gynecol 14:271-274

15. Peterson HB, Zhisen X, Hughes JM et al (1996) The risk of pregnancy after tubal sterilization: findings from the U.S. Collaborative Review of Sterilization. Amer J Obstet Gynecol 174:1161-1170

16. Zinaman MJ, Clegg ED, Brown CC et al (1996) Estimates of human fertility and pregnancy loss. Fertil Steril 65:503-509 